\title{
Micromachined glass apertures for artificial lipid bilayer formation in a microfluidic system
}

\author{
Mairi E Sandison, Michele Zagnoni, Mustafa Abu-Hantash and \\ Hywel Morgan
}

School of Electronics and Computer Science, University of Southampton, Southampton, UK

E-mail: mes@ecs.soton.ac.uk

Received 2 January 2007, in final form 2 May 2007

Published 2 July 2007

Online at stacks.iop.org/JMM/17/S189

\begin{abstract}
The use of spark assisted chemical engraving (SACE) to produce glass apertures that are suitable for the formation of artificial bilayer lipid membranes is described. Prior to use, the glass apertures were rendered hydrophobic by a silanization process and were then incorporated into a simple microfluidic device. Successful bilayer lipid membrane (BLM) formation and the subsequent acquisition of single-channel recordings are demonstrated. Due to the simplicity and rapidity of the SACE process, these glass apertures could be easily integrated into an all-glass microfluidic system for BLM formation.
\end{abstract}

\section{Introduction}

In recent years, several research groups have begun developing microfabricated systems for the formation of artificial bilayer lipid membranes (BLMs), into which membrane proteins can be subsequently reconstituted [1-3]. BLMs act as a natural support for membrane proteins and may be formed by the self-assembly of a lipid-solvent film that is spread across a hydrophobic aperture, surrounded by an aqueous medium. Artificial BLMs created in this manner have been in use for over 40 years [4]. As well as being required for fundamental studies of protein function using standard electrophysiological techniques, they are of importance for a wide range of applications, including the development of high sensitivity biosensors, drug screening platforms and single molecule protein studies $[5,6]$.

To exploit the many benefits of miniaturization (such as the small quantities of solution required by microfluidic systems, the potential for developing high-throughput analytical platforms and the integration of electrical and optical analytical techniques), we and other groups have previously developed both silicon and poly(methyl methacrylate) microdevices for forming suspended lipid bilayers [7-12]. Whilst there are several difficulties associated with using silicon devices, including the fragility of silicon nitride membranes and problems due to the low resistivity of silicon or large shunt capacitances, polymeric devices overcome these issues and provided a rapid, low cost solution to fabrication.

One major drawback of these polymeric devices, however, is their lack of chemical resistance, meaning that they are essentially single-use devices. In situations where it would be desirable to thoroughly clean a device before use (for instance, when performing single molecule fluorescence experiments) or to use the same device more than once (so as to ensure constant geometric parameters), an all-glass design would be advantageous, due to the excellent chemical resistance and transparency of glass, and its ability to withstand hot solutions and ultrasonication when cleaning. Furthermore, glass devices would also be less prone to scratching and would be more robust.

The formation of BLMs across microfabricated glass apertures has previously been reported, as has the acquisition of G $\Omega$ seal resistances when performing patch clamp experiments across such apertures [13, 14]. However, in these examples, an ion track etching method was employed to create the apertures. This involved firing a single gold ion through a thinned region $(80 \mu \mathrm{m})$ of a glass substrate and then etching down the latent ion track using hydrofluoric acid. Whilst this approach produced smooth, well-defined, micronscale apertures, it required the use of a linear accelerator, an extremely expensive piece of equipment that is not widely available. This paper presents an alternative approach, where 
a simple spark assisted chemical engraving (SACE) process (also known as electrochemical discharge machining, ECDM) is used to form glass apertures that are subsequently silanized to produce a suitably hydrophobic surface for BLM formation.

SACE is a type of electric discharge machining technique that is used to pattern non-conductive samples, which are immersed in an electrolyte solution. It was first reported as a machining technique by Kurafuji et al in 1968 [15], who employed it for drilling holes through glass workpieces, and has since been employed to create a variety of microstructures in glass [16-18] and other materials. Typically, the work piece is submerged in a strong electrolyte solution (such as $10-30 \% \mathrm{NaOH}$ ) directly beneath a tool electrode (generally with a gap of less than $25 \mu \mathrm{m}$ [19]). A potential of 25-50 V is applied between the tool electrode (cathode) and a counter electrode (anode), resulting in electrolysis at both electrodes, with $\mathrm{H}_{2}$ being generated at the cathode and $\mathrm{O}_{2}$ at the anode. If the applied voltage is above the critical voltage of the system (which is dependent upon the electrolyte concentration and the tool electrode material and geometry [16]), the rate of electrolysis at the tool electrode will be high enough to produce such a density of bubbles that they coalesce to form a gas film, thus separating the electrode from the electrolyte [19, 20]. The electric field in this gas film will be sufficiently high to cause electrical discharges from the electrode to the electrolyte. A combination of chemical reactions (involving the complexation of silicate) and thermal effects resulting from the discharge results in etching of the workpiece in the vicinity of the tool electrode.

Here we combine the SACE process with glass silanization to produce a simple and robust alternative to $\mathrm{Si}$ and polymer apertures for the creation of suspended BLMs. By incorporating the glass apertures into a simple microfluidic device, the formation of suspended BLMs that are amenable to both optical and low noise electrical measurements is demonstrated. Furthermore, the SACE technique is readily applicable to creating apertures through microfluidic channels that have previously been structured using an alternative glass etching technique, thereby enabling the production of an all-glass microfluidic device for suspended BLM formation. Such a device would be low-cost, robust, and chemically and thermally resistant, with favourable electrical and optical properties, thus combining the benefits of both silicon and polymeric devices.

\section{Materials and methods}

\subsection{Formation of apertures by SACE}

The SACE apparatus employed for machining apertures is described in figure 1. A function generator outputting a $5 \mathrm{~Hz}$ square wave was used to gate a FET connected to the output of a power supply. The sample, counter and tool electrodes were immersed in a $30 \%(\mathrm{w} / \mathrm{v}) \mathrm{NaOH}$ solution, the tool electrode being attached to a Z-positioner, whilst the entire SACE cell was mounted on an $X-Y$ stage over a microscope objective, enabling the etching process to be monitored. Initially, the tool electrode was positioned slightly above the sample surface (30-40 $\mu \mathrm{m}$ higher), so that no etching occurred until the electrode was lowered further [19]. Then, after a $30 \mathrm{~V}$ square

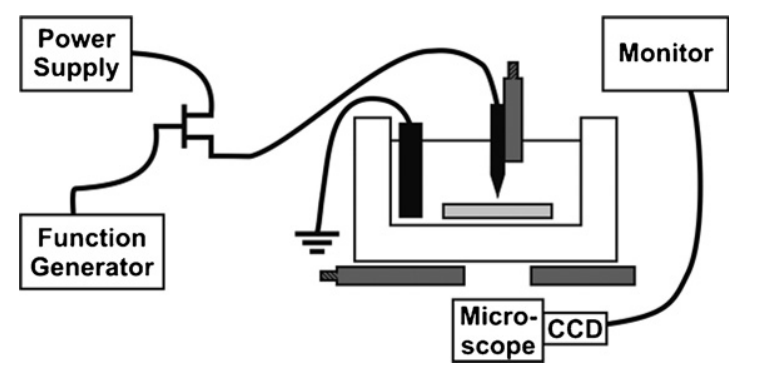

Figure 1. SACE apparatus. The sample (light grey) and the counter and tool electrodes (black) are immersed in a NaOH bath. The bath is mounted on an $X-Y$ stage (dark grey, with a central hole for a microscope objective, the microscope being connected to a CCD camera), so that the sample can be positioned with respect to the tool electrode. The latter is attached to a Z-positioner (dark grey).

wave had been applied, the electrode was lowered downwards in one step until it was approximately $10 \mu \mathrm{m}$ above the surface. From this point onwards, the tool electrode was gradually and continuously lowered until bubbles were observed rushing along the bottom of the sample, indicating that an aperture had formed. The voltage supply was then cut off and the total $z$-movement and machining time (from the initial $10 \mu \mathrm{m}$ position onwards) were noted.

When forming BLMs, an aperture diameter in the range of $50-150 \mu \mathrm{m}$ is typically desirable. In order to achieve this, a 3.5-5 $\mu \mathrm{m}$ tip-diameter tungsten probe (SE-20T or SE-T Signatone probes, Sel-Tek, Glasgow) was employed as the tool electrode and apertures were machined in 100-160 $\mu \mathrm{m}$ thick borosilicate glass coverslips. In addition, to assemble a glass aperture within a fluidic device, inlet and outlet holes were also required (as discussed below and as illustrated in figure 2). These were machined using a $150 \mu \mathrm{m}$ diameter stainless steel wire.

\subsection{Silanization of glass apertures}

To create a sufficiently hydrophobic surface for BLM formation, the glass apertures were chemically modified using a silane compound, perfluorooctyl-trichlorosilane (PF-TCS). Before silanization, the glass apertures were cleaned in an ultrasonic bath with the following sequence of solutions: 1:5 Decon 90 in $\mathrm{RO}$ water at $60{ }^{\circ} \mathrm{C}, \mathrm{RO}$ water, acetone, methanol, $\mathrm{RO}$ water. Two procedures were then employed to silanize the glass surface. The first was to immerse the sample for $30 \mathrm{~min}$ in a $1 \%$ silane solution diluted with ethanol, before thoroughly rinsing it with ethanol and then blow drying. The second was to place the sample in a vacuum chamber alongside $50 \mu \mathrm{l}$ of undiluted silane prior to evacuating the chamber until the silane droplet began to bubble off. The sample was then left exposed to the silane vapour for $30 \mathrm{~min}$. In both cases, the glass aperture was subsequently baked at $120^{\circ} \mathrm{C}$ for $20 \mathrm{~min}$. To assess the hydrophobicity of the silanized glass, a simple microscope was used to visualize the contact angle of a droplet of water, approximately $0.5 \mu \mathrm{l}$ in volume.

\subsection{Microfluidic devices for artificial BLM formation}

Figure 2 illustrates the structure of a useable microfluidic device for BLM formation. It can be envisaged fabricating 

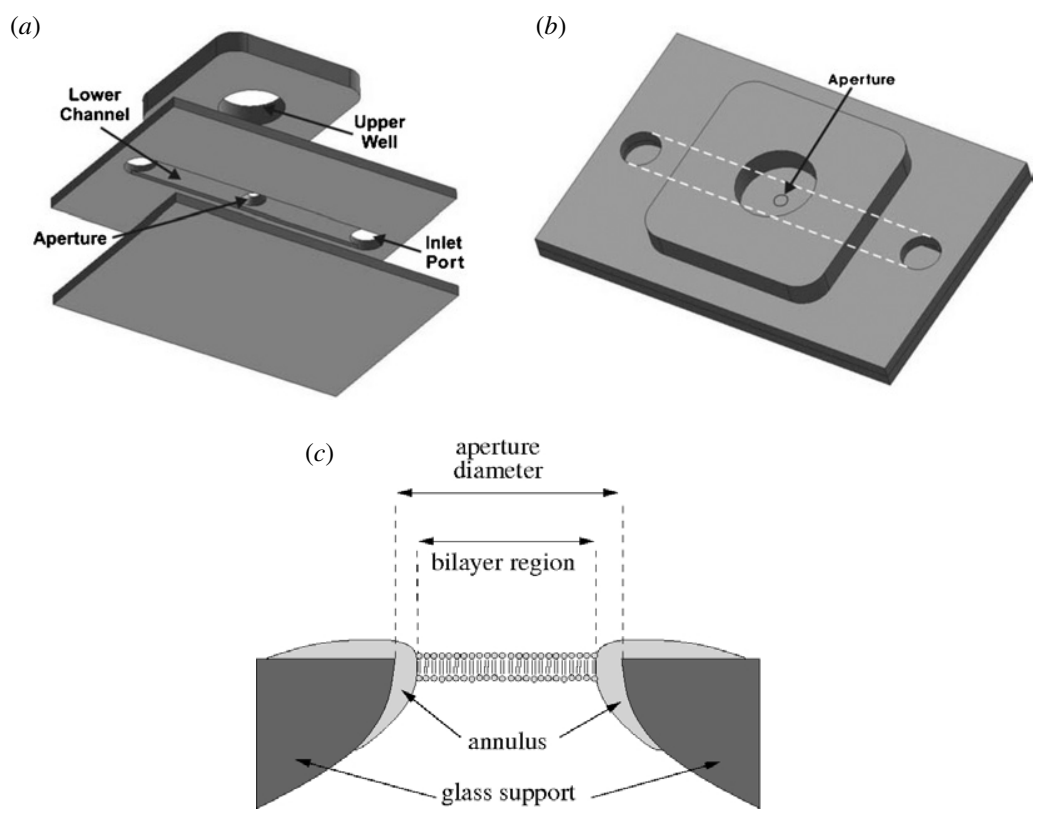

Figure 2. An all-glass microfluidic device for BLM formation. Diagrams illustrating a microfluidic structure that can be employed to create on-chip lipid bilayers. It incorporates a central aperture that is situated between a lower microfluidic channel and an upper open well. ( $a$ ) An exploded view of the structure, looking from beneath. $(b)$ The complete structure from above, with the edges of the embedded lower channel marked by the white dashed lines. (c) An illustration depicting an artificial lipid bilayer formed across a glass aperture. The bilayer region, which self-assembles from a lipid solution that is applied to the aperture, is surrounded by a thicker lipid-solvent annulus.

such a structure by a combination of wet etching, SACE and glass thermal bonding (as will be discussed below). However, for assessing the suitability of silanized glass apertures for the formation of suspended BLMs, a simple microfluidic test device with the same structure can be very rapidly constructed using a double sided adhesive tape to form fluidic channels.

These test devices comprised (from the base of the structure upwards): a glass coverslip, a layer of $100 \mu \mathrm{m}$ thick double sided adhesive that had a 1-2 mm wide channel cut out of it; the silanized glass coverslip with its central aperture and two larger holes for fluidic access, corresponding to both ends of the lower channel; a PMMA well that was bonded to the silanized glass using a cyanoacrylate adhesive; and an inlet and an outlet reservoir that were attached over the two ports using epoxy (not shown in figure 2). The aperture was aligned so as to be in the centre of both the fluidic channel and the PMMA well.

\subsection{Formation of on-chip artificial BLMs}

When in use, the device was mounted over the $\times 40$ objective of a transmission microscope that was connected to a CCD camera. Before attempting to form a BLM, the lower channel was filled with buffer solution (typically $0.15 \mathrm{M} \mathrm{KCl}$ ), and $\mathrm{Agl} \mathrm{AgCl}$ electrodes were inserted into the lower channel inlet and into the well. These electrodes were attached to an ID562 BLM amplifier (Industrial Developments Bangor, UK) that was connected, via a National Instruments $6036 \mathrm{E}$ data acquisition card (Newbury, UK) to a PC. $0.2 \mu \mathrm{l}$ of $20 \mathrm{mg} \mathrm{ml}^{-1}$ 1,2-diphytanoyl-sn-glycero-3-phospho-choline in n-decane was then injected onto the base of the well. After waiting a few seconds for the lipid solution to flow over the silanized glass, buffer was injected into the upper well.
If a bilayer did not form spontaneously (as determined from capacitance measurements and by optically monitoring the aperture area), the buffer in the upper well was either agitated or removed, and re-injected so as to encourage the thinning process. To confirm that a BLM had successfully formed and to assess the current noise, a solution of Gramicidin D (Sigma-Aldrich, UK) was injected into the upper well.

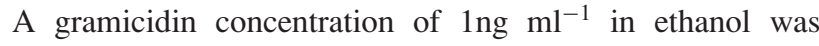
employed, and $1 \mu \mathrm{l}$ was injected.

\section{Results and discussion}

\subsection{Formation of apertures by SACE}

Using the procedure described above, glass apertures with diameters in the desired range were successfully produced by the SACE process. By using a tungsten probe with a small tip diameter $(3.5-5 \mu \mathrm{m})$ as the tool electrode and by carefully controlling the descent of the electrode using a micromanipulator (something that cannot be done using a gravity feed system), a range of apertures with different dimensions were produced. For instance, in order to produce an aperture with a diameter of approximately $100 \mu \mathrm{m}$ through a $100 \mu \mathrm{m}$ thick glass coverslip, the tool electrode was moved downwards at a continuous rate, from $10 \mu \mathrm{m}$ above the surface to $80 \mu \mathrm{m}$ below over a period of $30 \mathrm{~s}$. By varying the rate at which the tool electrode is lowered, its final vertical position and the length of time between the emergence of the initial aperture opening and the shutting off of the power supply, different aperture diameters and aspect ratios may be obtained using the same tool electrode: the faster the 

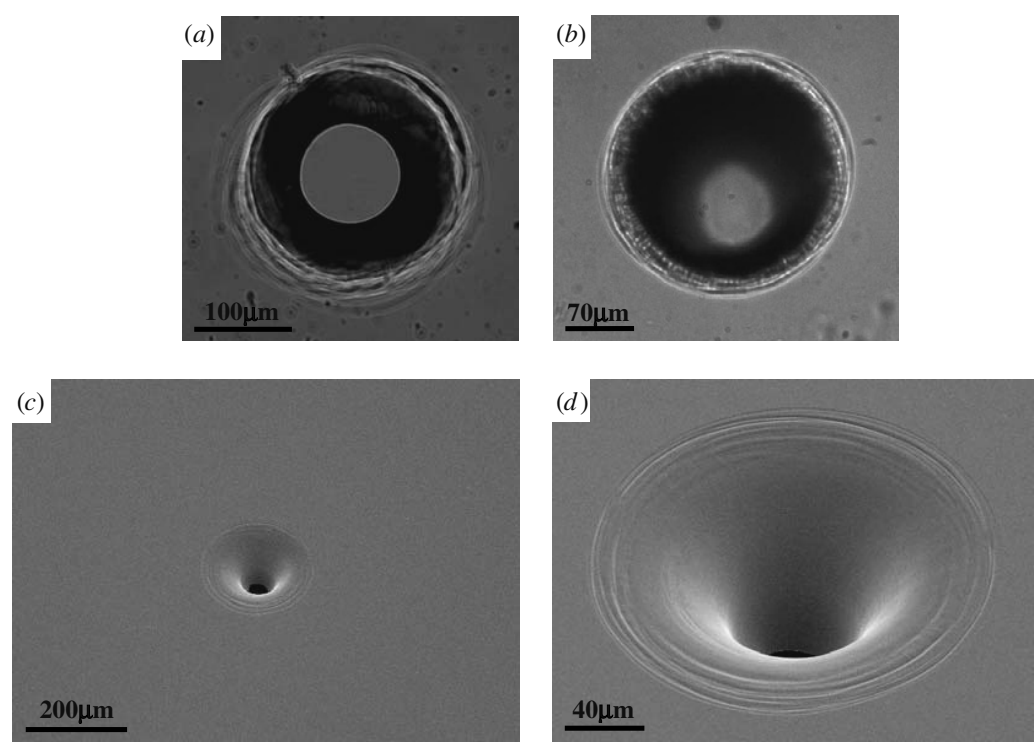

Figure 3. Glass apertures formed by SACE. Optical microscope images of a $100 \mu \mathrm{m}$ diameter aperture focused on the narrow opening (a) and a $70 \mu \mathrm{m}$ diameter aperture focussed on the wider opening $(b)$. Electron micrographs of a $40 \mu \mathrm{m}$ diameter aperture showing that no damage occurs to the surrounding glass and that smooth side walls are obtained $(c$ and $d$ ).

descent and the faster the cut-off, the shallower and smaller the resulting aperture. Other parameters such as electrode dimensions, temperature, electrolyte concentration, applied voltage and current limitation were not varied during this work but these factors have been well studied elsewhere and could be optimized if required [16-20].

As can be seen from figure 3, the resulting apertures have a conical shape, with the tool electrode side being significantly wider, in the order of 200-250 $\mu \mathrm{m}$ diameter in these examples. (This taper results primarily from the isotropic nature of the etching process, so the upper entrance side, which will undergo some etching throughout the entire machining process, will have a diameter that is larger than the lower side.) This shape is actually beneficial for bilayer experiments, as it will lower the access resistance of the system [21]. Three apertures with different narrow-side diameters are shown: $100 \mu \mathrm{m}$, $70 \mu \mathrm{m}$ and $40 \mu \mathrm{m}$. It can be seen that there is no damage to the surrounding glass and that the sidewalls of the apertures are very smooth. There are some undulations around the wider opening (shallow ring patterns can be clearly seen in the SEMs), but as the BLMs are to be formed across the narrower side of the aperture, this is not important. Additionally, the SACE process is a very rapid method of creating apertures, the total machining time for each of these apertures being less than 1 min.

Furthermore, whilst these apertures have favourable dimensions for BLM formation, the production of apertures with decreased dimensions should be readily achievable. Improvements that could be incorporated in order to achieve this include automation of the $z$-movement, so as to precisely control the speed of the tool electrode, and integration of a system that enables more rapid shutdown of the voltage after the etching process initially opens up the narrow side of the aperture. Of course, the use of a smaller tool electrode with a shallower cone angle would also be beneficial ${ }^{1}$. With such modifications, it should be possible to rapidly create significantly smaller glass apertures, potentially within the region of patch clamp requirements, where an aperture no more than a few microns in diameter is required [22].

A single tool electrode (figure $4(a)$ ) can be employed to machine several apertures, although it will degrade slightly with use. Figure $4(b)$ shows an electrode tip that has been used to machine 15 samples, the degradation resulting from a combination of erosion and mechanical damage from contacting the work piece. Corresponding images of an aperture machined by this electrode are given in figure $4(d)$, the contour of the aperture being more elongated and slightly uneven.

Tool electrodes, however, can be re-generated using an electrochemical sharpening process, electrochemical techniques having been widely employed for creating sharp probes for scanning tunnelling microscopy (STM) and atomic force microscopy (AFM) [17]. Indeed, these techniques could be employed to create the tool electrode from a plain metal wire, which would enable some degree of tailoring to a desired electrode shape. (This may be beneficial if attempting to produce smaller features or to create a variety of sidewall angles.) When regenerating a tool electrode for BLM aperture formation, all that is required is a brief $(5-10 \mathrm{~s})$ application of an inverse SACE potential to the electrode (the tool electrode becoming the anode) in the same $\mathrm{NaOH}$ solution, which induces oxidative dissolution of the tungsten

1 It has previously been reported that, even when using extremely sharp probes as the tool electrode (with apex radii of $<100 \mathrm{~nm}$ ), a minimum feature size of $25 \mu \mathrm{m}$ is attainable [17]. However, this refers to the tool-side width of the feature and not to the dimensions of its base, which are noticeably narrower. As, for BLM aperture formation, it is the narrowest side that is important, it should be possible, using sharp electrodes and careful control of the voltage shutdown after opening up the lower side, to create significantly smaller apertures. 

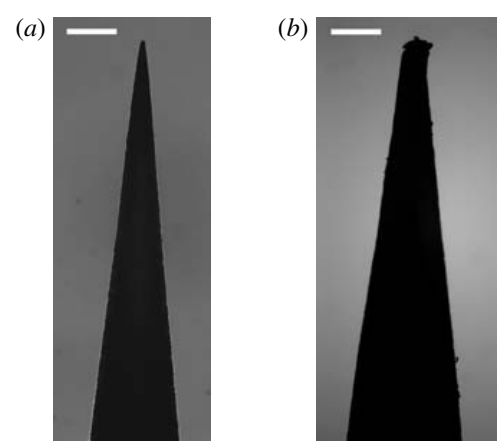

(c)

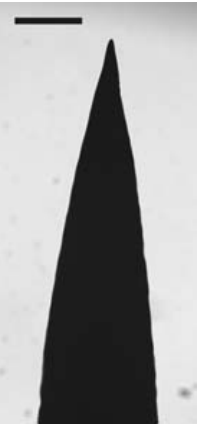

(d)

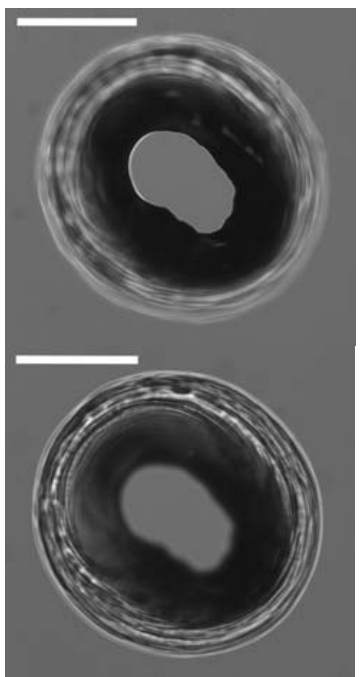

(e)

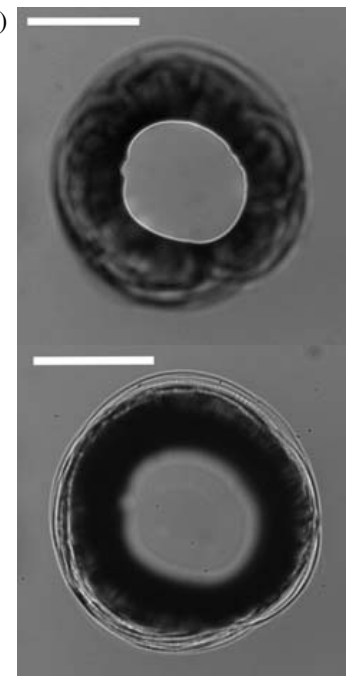

Figure 4. SACE tool electrodes. (a) An unused tool electrode. (b) The same tool electrode after machining 15 apertures, which now has a rougher, non-circular tip. (c) A tool electrode after regeneration. (d) An aperture formed with the electrode shown in (b), the upper image focused on the narrower opening and the lower image on the wider side. (e) An aperture formed with a regenerated electrode (upper and lower images as before). In all pictures, the scale bar is $100 \mu \mathrm{m}$.

probe (figure $4(c)$ ). Although the resulting shape is not as sharp and well defined as the original, commercial probe (as the regeneration procedure employed, whilst rapid and simple, has not been fully optimized), it is nonetheless satisfactory for producing apertures for BLM formation, as shown in figure 4(e). Hence, one tool electrode can be employed many times and may be effectively regenerated without having to remove it from the SACE bath, which is useful if the formation of multiple apertures is required.

\subsection{Silanization of glass apertures}

Before the glass apertures can be employed for BLM formation they must be made hydrophobic. This is due to the bilayer formation process relying upon surface-tensioninduced drainage of the non-polar solvent (decane in this case) to thin the initially thick lipid-solvent layer down to that of a lipid bilayer. Silane coupling agents are widely used for modifying the surface chemistry of glass substrates [23], as the silane anchor group (which couples to surface hydroxyl groups via siloxy linkages) can be attached to a wide variety
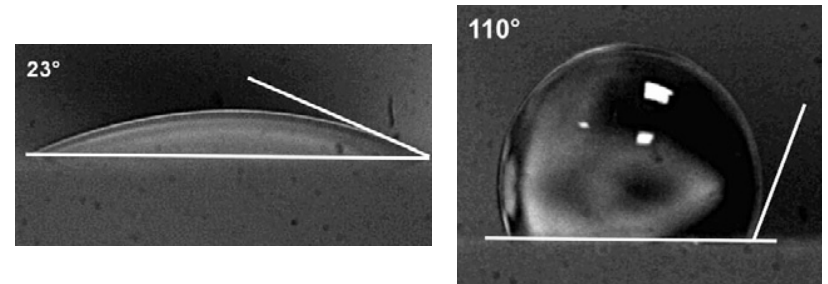

Figure 5. Contact angle of glass surfaces. Images showing the contact angle of a native glass surface (left) and a glass sample silanized by vapour-deposition of PF-TCS (right).

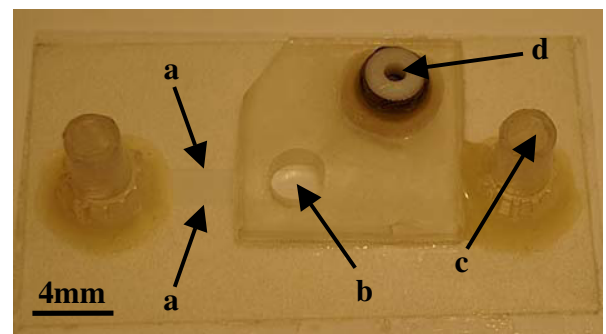

Figure 6. A microfluidic test device. A microfluidic device used to test the formation of bilayers across silanized glass apertures. The boundaries of the lower channel $(a)$ and the position of the aperture (b) are marked. One electrode was inserted into an inlet reservoir for the lower channel $(c)$ and the other into the upper well. In this example, a channel machined into the PMMA connected the base of the upper well to a fluidic connector $(d)$, so as to enable simple manipulation of the solution within the well.

(This figure is in colour only in the electronic version)

of functional groups. In PF-TCS, the silane group is linked to a hydrophobic, fluorinated carbon chain. By functionalizing the surface of the glass apertures with PF-TCS, using either of the two procedures described above, a significant increase in the measured contact angle was obtained (figure 5). Clean, unmodified glass exhibits a contact angle in the order of 20-25 for de-ionised water. After silanization, the surface of glass became markedly hydrophobic and the contact angle rose to approximately $110^{\circ}$ (close to that of Teflon ${ }^{\circledR}$, whose contact angle was measured to be in the order of $114^{\circ}$ ).

Unlike some previous reports regarding silanized surfaces for suspended BLM supports [12], the PF-TCS coating proved to be quite durable and it was not necessary to silanize the apertures on the day of BLM formation. When the contact angle of silanized glass samples was measured after $24 \mathrm{~h}$, $48 \mathrm{~h}, 10$ days, 30 days and 90 days of storage (in air at room temperature), a negligible difference in contact angle was observed with respect to the initial value, even after 90 days (when the value was measured as approximately $108^{\circ}$ ). Therefore, multiple apertures may be silanized at one time and stored for later use.

\subsection{Formation of on-chip artificial BLMs}

An example of a test device used for assessing the suitability of silanized glass apertures for BLM formation is shown in figure 6. 
Forming stable BLMs across the silanized glass apertures proved to be relatively straightforward. Following injection, the lipid solution was observed to flow rapidly outwards over the glass surface. Occasionally, shortly after the addition of buffer solution, this initially thick lipid-solvent film spontaneously thinned to form a BLM. More typically, however, agitation, or removal and re-injection of the solution in the upper well was required to induce the thinning process (as in figure 7(a)). The thinning of the lipid film was observed both optically (figure $7(b)$ ) and electrically ${ }^{2}$ (by monitoring the capacitance between the electrodes and comparing it to the value expected for a BLM, which was calculated from the aperture area assuming a specific membrane capacitance of approximately $\left.0.5 \mu \mathrm{F} \mathrm{cm} \mathrm{cm}^{-2}[3,9]\right)$.

The background currents obtained were low, with a current noise of less than $1 \mathrm{pA}$ for an applied potential of $100 \mathrm{mV}$ at $1 \mathrm{kHz}$, and the BLMs could typically withstand a voltage of $250-300 \mathrm{mV}$. In order to demonstrate single channel recording and to verify the formation of a bilayer, the transmembrane peptide gramicidin was incorporated into a BLM. Gramicidin D is a heterogeneous mixture of six linear pentadecapeptide components from Bacillus Brevis that forms ion channels specific for monovalent cations [24]. Gramicidin monomers, which diffuse within a single leaflet of a lipid bilayer, can temporarily come together to form dimmers that act as pores, resulting in ion conduction through the bilayer [25]. As these ion channels cannot span a lipid film that is thicker than a bilayer, they can be employed to confirm BLM formation. Traces showing ion conduction through gramicidin channels in a BLM, formed within a microfluidic test device, are shown in figure 8 . Clear, quantized jumps can be seen for both lower and higher numbers of channels (with a single channel event being in the order of 1-2 pA).

\subsection{Incorporation into a microfluidic device}

As it requires no etch mask and as position control is straightforward, the SACE process for aperture creation could be employed in conjunction with other rapid microfabrication techniques for the production of a simple, all-glass microfluidic platform for BLM formation (with a structure similar to that of figure 2(a)). A microfluidic channel could be defined by either a wet etching process, such as the simple procedure outlined in [8], or by alternative techniques, such as sandblasting [26], which circumvent the need for HF etchants (although the resulting channel surface will be significantly rougher than in the former case). An aperture could then be created through the centre of the channel by SACE. To

2 Lipid bilayers are typically formed by self-assembly of an initially thick lipid-solvent film, which will have a monolayer of amphipathic lipid molecules at each of its aqueous interfaces [23]. As previously noted, thinning of the lipid-solvent film arises primarily from the wetting of the hydrophobic support by the organic solvent. Since the lipid-solvent film may be considered as a dielectric layer in a parallel plate capacitor, the thinning of the lipidsolvent film from a thick layer down to that of a lipid bilayer ( $\sim 5 \mathrm{~nm}$ thick) may be monitored by measuring the capacitance across the aperture. The measured capacitance will increase as the lipid-solvent layer thins, reaching a stable value once a BLM spans the majority of the aperture, with a thicker lipid-solvent annulus (known as the Plateau-Gibbs border) remaining around the perimeter of the aperture. BLM formation may also be monitored optically. When using a transmission microscope, the emergence of a bilayer region will be observed as an expanding lighter region within the aperture, the annulus region remaining darker.
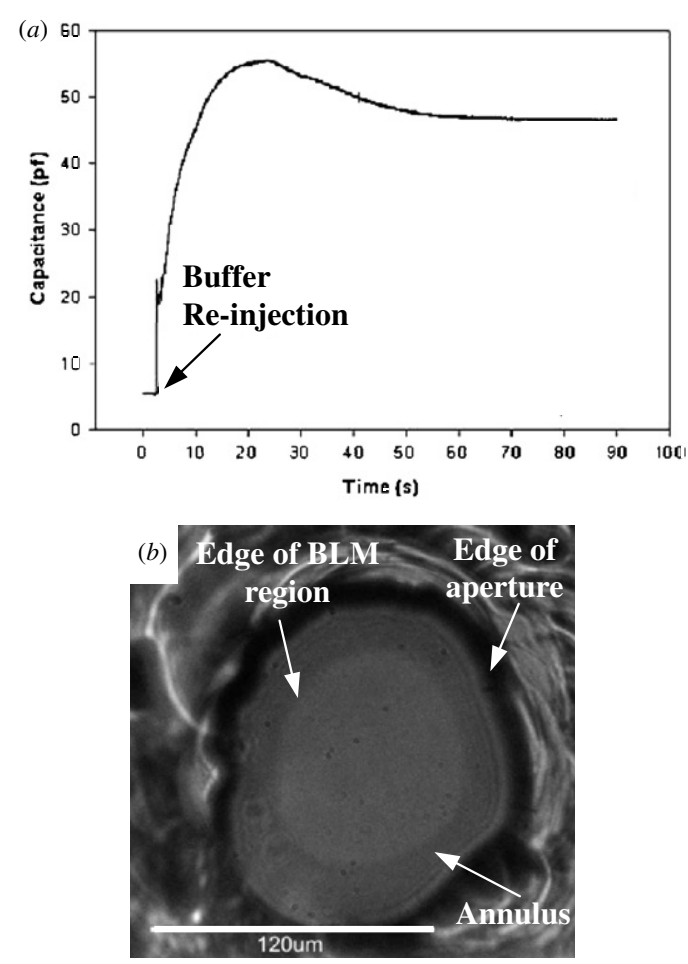

Figure 7. BLM formation and image. (a) A capacitance trace acquired during formation of a bilayer within a $120 \mu \mathrm{m}$ diameter aperture, which stabilized at a value of $47 \mathrm{pF}$. A capacitance in the order of $45-50 \mathrm{pF}$ would be expected for this aperture, when taking into account the presence of a surrounding annulus and assuming a specific membrane capacitance of approximately $0.5 \mu \mathrm{F} \mathrm{cm}^{-2}$. In this case, the BLM did not form spontaneously after buffer addition, so the solution in the upper well was removed and then re-injected in order to encourage thinning of the lipid film (the recording of this trace started prior to buffer re-injection). Although the bilayer region initially expanded to fill the entire aperture, it then began to shrink back slightly, creating a more stable structure with a wider supporting annulus (a behaviour that is occasionally observed). (b) An image of the aperture region after BLM formation, the light central area being the BLM region. This light area was seen to emerge as the lipid-solvent film within the aperture began to thin down to form a BLM.

seal the channel with a smooth substrate, a rapid and lowtemperature bonding process, such as that previously described for soda-lime glass bonding [27] could then be employed. (A slightly modified version of this process also proved successful when bonding soda-lime to the borosilicate glass, the process employed being the following: after clamping the two glass pieces together, they were placed in an oven at $50{ }^{\circ} \mathrm{C}$, ramped to $500^{\circ} \mathrm{C}$ at approximately $8^{\circ} \mathrm{C} \mathrm{min}^{-1}$, held at this temperature for $30 \mathrm{~min}$, before raising it to $575^{\circ} \mathrm{C}$, then held there for a further $2 \mathrm{~h}$, before finally cooling the oven down overnight to $50{ }^{\circ} \mathrm{C}$.)

By employing thinnest obtainable borosilicate and sodalime substrates for the fabrication of the device (the thinnest sheets being available in borosilicate), the resulting structure would enable a BLM in the aperture to be visualized through a high NA, high magnification objective. This would allow for the acquisition of both single molecule fluorescence data, using for instance conventional wide-field epifluorescence microscope [28] and simultaneous electricaloptical measurements. Additionally, the fabrication process 

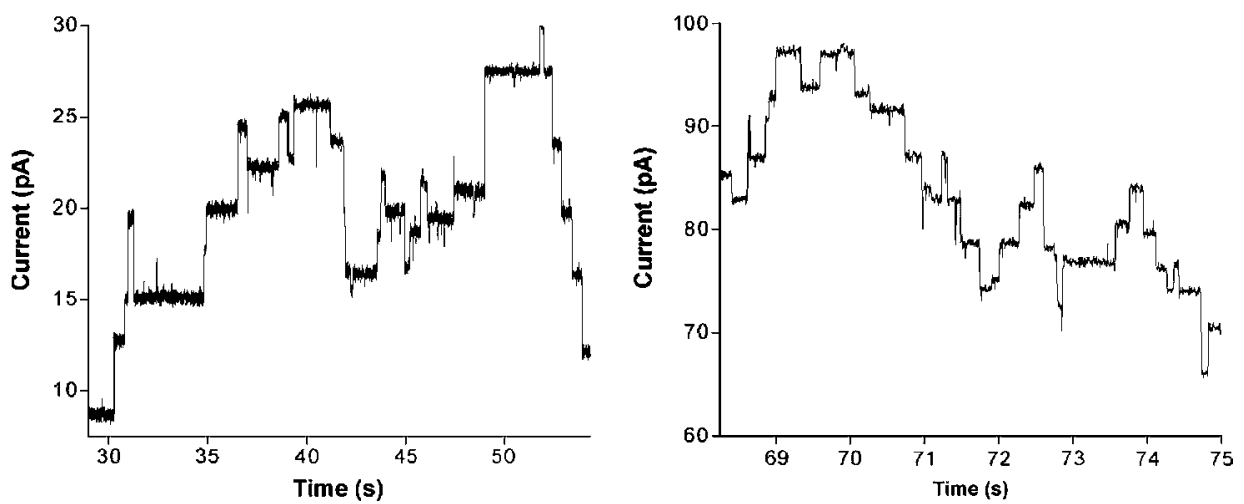

Figure 8. Single channel conductances of gramicidin. Traces showing the activity of gramicidin ion channels reconstituted into a BLM suspended across a glass aperture in a microfluidic device. The second trace (with the smaller time and larger current scales) was recorded approximately $10 \mathrm{~min}$ after the first, by which point many more gramicidin molecules would have incorporated into the BLM. These traces were obtained at $100 \mathrm{mV}, 1 \mathrm{kHz}$ in $150 \mathrm{mM} \mathrm{KCl}, 10 \mathrm{mM}$ Hepes, $\mathrm{pH} 7.4$ buffer and were then digitally filtered at $200 \mathrm{~Hz}$. Additionally, the BLM was formed using a 7:3 POPE:POPG (w/w) lipid solution.

would be amenable to up-scaling for the production of a lowcost, robust, chemically resistant BLM array platform.

\section{Conclusion}

The feasibility of using SACE followed by silanization to create apertures that are suitable for BLM formation has been demonstrated. The resulting apertures were smooth and a variety of diameters were obtained. BLMs were successfully formed within glass apertures that were incorporated into a simple microfluidic device. This straightforward fabrication technique could be readily employed in conjunction with other well-established processes to create an all-glass microfluidic device for artificial BLM studies.

\section{Acknowledgments}

This work was supported by the UK Interdisciplinary Research Centre in Bio-Nanotechnology (R45659/01) and the 6th Framework Programme of the European Commission under the contract NMP4-CT-2005-017114 'RECEPTRONICS'.

\section{References}

[1] Eray M, Dogan N S, Liu L J, Koch A R, Moffett D F, Silber M and Vanwie B J 1994 Highly stable bilayer-lipid membranes (BLMs) formed on microfabricated polyimide apertures Biosensors Bioelectron. 9 343-51

[2] Schmidt C, Mayer M and Vogel H 2000 A chip-based biosensor for the functional analysis of single ion channels Angew. Chem. Int. Ed. 39 3137-40

[3] Cheng Y, Bushby R J, Evans S D, Knowles P F, Miles R E and Ogier S D 2001 Single ion channel sensitivity in suspended bilayers on micromachined supports Langmuir 17 1240-2

[4] Mueller P, Rudin D O, Tien H T and Wescott W C 1962 Reconstitution of cell membrane structure in vitro and its transformation into an excitable system Nature $194979-80$

[5] Bayley H and Cremer P S 2001 Stochastic sensors inspired by biology Nature $143226-30$

[6] Ide T and Yanagida T 2002 Development of an experimental apparatus for simultaneous observation of optical and electrical signals from single ion channels Single Mol. $333-42$

[7] Morgan H, Sandison M E, Mendes G, Berry R and Watts A 2004 Artificial lipid bilayers in a microfabricated system Proc. $\mu$ TAS 2004, 8th Int. Conf. on Miniaturized Systems for Chemistry and Life Sciences vol 1 pp 330-2

[8] Sandison M E and Morgan H 2005 Rapid fabrication of polymer microfluidic systems for the production of artificial lipid bilayers J. Micromech. Microeng. 15 S139-44

[9] Suzuki H, Tabata K, Kato-Yamada Y, Noji H and Takeuchi S 2004 Planar lipid bilayer reconstitution with a micro-fluidic system Lab Chip 4 502-5

[10] Suzuki H, Tabata K V, Noji H and Takeuchi S 2006 Highly reproducible method of planar lipid bilayer reconstitution in polymethyl methacrylate microfluidic chip Langmuir 22 1937-42

[11] Peterman M C, Ziebarth J M, Braha O, Bayley H, Fishman H A and Bloom D M 2002 Ion channels and lipid bilayer membranes under high potentials using microfabricated apertures Biomed. Microdev. 4 231-6

[12] Pantoja R, Sigg D, Blunck R, Bezanilla F and R Heath J 2001 Bilayer reconstitution of voltage-dependent ion channels using a microfabricated silicon chip Biophys. J. 81 2389-94

[13] Fertig N, Meyer Ch, Blick R H, Trautmann Ch and Behrends J C 2001 Microstructured glass chip for ion-channel electrophysiology Phys. Rev. E 64040901

[14] Fertig N, Klau M, George M, Blick R H and Behrends J C 2002 Activity of single ion channel proteins detected with a planar microstructure Appl. Phys. Lett. 81 4865-7

[15] Kurafuji H and Suda K 1968 Electric discharge drilling of glass Ann. CIRP 16415

[16] Lee E S, Howard D, Liang E, Collins S D and Smith R L 2004 Removable tubing interconnects for glass-based micro-fluidic systems made using ECDM J. Micromech. Microeng. 14 535-41

17] Wüthrich R, Hof L A, Lal A, Fujisaki K, Bleuler H, Mandin P and Picard G 2005 Physical principles and miniaturization of spark assisted chemical engraving (SACE) J. Micromech. Microeng. 15 S268-75

[18] Fascio V, Wuthrich R and Bleuler H 2004 Spark assisted chemical engraving in the light of electrochemistry Electrochim. Acta 49 3997-4003

[19] Wüthrich R and Fascio V 2005 Machining of non-conducting materials using electrochemical discharge phenomenon-an overview Int. J. Mach. Tools Manuf. 45 1095-108

[20] Basak I and Ghosh A 1996 Mechanism of spark generation during electrochemical discharge machining: a theoretical 
model and experimental verification J. Mater. Process. Technol. 62 46-53

[21] Mayer M, Kriebel J K, Tosteson M T and Whitesides G M 2003 Microfabricated Teflon membranes for low-noise recordings of ion channels in planar lipid bilayers Biophys. J. 85 2684-95

[22] Klemic K G, Klemic J F, Reed M A and Sigworth F J 2002 Micromolded PDMS planar electrode allows patch clamp electrical recordings from cells Biosensors Bioelectron. 17 597-604

[23] Ruckenstein E and Li Z F 2005 Surface modification and functionalization through the self-assembled monolayer and graft polymerization Adv. Colloid Interface 113 43-63

[24] Fujiwara H, Fujihara M and Ishiwata T 2003 Dynamics of the spontaneous formation of a planar phospholipid bilayer: a new approach by simultaneous electrical and optical measurements J. Chem. Phys. 119 6768-75
[25] Burkhart B M, Gassman R M, Langs D A, Pangborn W A and Duax W L 1998 Heterodimer formation and crystal nucleation of Gramicidin D Biophys. J. 75 2135-46

[26] Andersen O S, Koeppe II R E and Roux B 2005 Gramicidin channels IEEE T Nanobiosci. 4 10-20

[27] Yagyu H, Sugano K, Hayashi S and Tabata O 2005 Micropowder blasting with nanoparticles dispersed polymer mask for rapid prototyping of glass chip J. Micromech. Microeng. 15 1236-41

[28] Keir R, Igata E, Arundell M, Smith W E, Graham D, McHugh C and Cooper J M 2002 SERRS. In situ substrate formation and improved detection using microfluidics Anal. Chem. 74 1503-8

[29] Sandison M E, Malleo D, Holmes D, Berry R and Morgan H 2005 Artificial bilayer lipid membranes (BLMs) on-chip for single molecule sensing Nanotechnology II; Proc. SPIE $\mathbf{5 8 3 8} 252-7$ 